\title{
Effect of hydrophilicity of polyaniline particles on their electrorheology: Steady flow and dynamic behaviour
}

\author{
Martin Stěnička ${ }^{*}$, Vladimír Pavlínek ${ }^{3}$, Petr Sáha ${ }^{3}$, Natalia V. Blinova ${ }^{\text {b }}$, Jaroslav Stejskal ${ }^{13}$, Otakar Quadrat $^{\text {b }}$
}

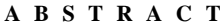

Electrorheological properties of suspensions are considerably affected by hydrophilicity of suspension particles. As a model material, polyaniline base powder protonated with sulfamic, tartaric, or perfluo- rooctanesulfonic acids provided particles of various hydrophilicity. The experiments revealed that, in the absence of electric field, due to a good compatibility of hydrophobic polyaniline particles with sili- cone-oil medium, their interactions were limited and the viscosity of suspension was low. When the electric field was applied, the rigidity of the polarized chain structure of the particles increased and, consequently, viscosity increased as well. In the contrast, the field-off suspension viscosity of highly interacting hydrophilic particles, which are incompatible with the oil, and where particle aggregation may set in, was high especially at low shear rates, and the material had a pseudoplastic character. Then, a relative increase in viscosity due to the polarization of the particles or their clusters in the electric field was much lower than in the former case. Due to a different primary structure of suspension, depending on the particle compatibility with the oil the field-off storage modulus of suspensions of hydrophobic particles was lower than the loss modulus, while in suspensions of hydrophilic particles the former modulus dominated. In both cases, an increase in elasticity with increasing electric field strength was higher than that in viscosity.
\end{abstract}

\section{Introduction}

The electrorheological (ER) phenomenon [1], an increase in viscosity of suspensions within milliseconds on application of a direct or alternating electric field, has been the object of a number of studies. The mechanism of building a fibrous or chain structure of polarized suspension particles organized along the electric stream-lines has been discussed in several review articles [2-8]. Due to its remarkable properties, this effect could be important in various engineering applications, such as in hydraulic valves, dampers and clutches. For that purpose, a strong viscosity increase within a short time interval is needed; hence particles of various materials suspended in the oil have been investigated. The performance of ER liquids, $e$, can be expressed as the ratio of electroviscosity [9], $\left.A f]_{E}=f\right]_{E}-r j_{0}$, and field-off viscosity, rjo, as e $=A f j_{E} l f j o t$ where $\%$ is the viscosity at the electric field strength used, E. It is obvious that not only $r j_{\mathrm{E}}$, but also $\left.r\right]_{0}$ is important in the selection of the material for practical use.

Our recent investigation of dynamic properties of silicone-oil suspensions of the silica particles surface-modified with urea during the transition from a liquid to solid state in the electric field showed that urea may strongly affect the field-off viscosity of suspensions [10]. It appeared that strong interactions of silica particles manifesting themselves by high suspension viscosity were eliminated by urea coating. These findings revealed that the primary structure of particle chains in the suspension controlled by compatibility of components may considerably influence the ER performance [11]. It is clear that a very high $r j_{E}[12]$ need not a priori mean a great relative increase in ER viscosity.

In the present study, suspensions of polyaniline (PANI) particles of various compatibility with the silicone oil have been investigated. The conducting PANI proved to be a suitable material due to its easy preparation. Its chemical structure given by alternating of a- and nbonds and the presence charge carriers allows controlling conductivity of PANI by treatment with various organic and inorganic acids to various extents [13-15]. Electric properties of PANI can also be influenced efficiently by changing of the reaction conditions during the polymerization of aniline [16,17]. Thus, the material with variable conductivity and dielectric properties [18-21] can be obtained, while the morphology and particle size of PANI remain virtually the same [15].

PANI base and acid constitute a salt. The mass fraction of acid in the salt is $10-50 \%$ [15], depending on the degree of protonation and molar mass of an acid. Therefore, the properties of acids are projected into those of salts. When acids having a long hydropho 
bic chain, such as perfluorooctanesulfonic acid, produce a salt with PANI base, perfluorinated chain endows resulting PANI salt with a marked hydrophobicity. When, on the contrary, an acid containing hydroxy or amino groups, such as in tartaric or sulfamic acids, respectively, is used for reprotonation of PANI base, PANI salt is considerably hydrophilic. For that reason, PANI represents a good model for ER studies because the particle hydrophilicity can be varied while keeping other properties about the same.

\section{Materials and methods}

\subsection{Preparation of PANI and its protonation}

PANI powder was prepared by the oxidation of $0.2 \mathrm{~mol} \mathrm{~L}^{-1}$ aniline hydrochloride with $0.25 \mathrm{~mol} \mathrm{~L}^{-1}$ ammonium peroxydisulfate in aqueous medium [22]. PANI hydrochloride obtained by the polymerization was converted to a PANI base with excess of $1 \mathrm{~mol} \mathrm{~L}^{-1}$ ammonium hydroxide. The conductivity of dry PANI base was $3 \times$ $10^{\text {“9 }} \mathrm{S} \mathrm{cm}$ “1. The PANI base $(1.81 \mathrm{~g}, 5 \mathrm{mmol}$; four aniline units considered as repeating units) were suspended in $40 \mathrm{~mL}$ of aqueous acid solution for $24 \mathrm{~h} \mathrm{[15].} \mathrm{The} \mathrm{resulting} \mathrm{(protonated)} \mathrm{PANI} \mathrm{salt} \mathrm{was}$ filtered off, rinsed with acetone, dried in air at room temperature and kept in a desiccator.

\subsection{PANI characterization}

In this study, four PANI samples (S1-S4) were used (Table 1). The water contact angles measured on compressed pellets were assessed with a measuring system OCA20 (Dataphysic, Germany). The conductivity was measured by a four-point van der Pauw method on the same pellets, $13 \mathrm{~mm}$ in diameter, compressed at $700 \mathrm{MPa}$ with a manual hydraulic press, using a current source SMU Keithley 237 and a Multimeter Keithley 2010 voltmeter with a 2000 SCAN 10-channel scanner card [15]. The highest contact angle of PANI particles protonated with perfluorooctanesulfonic acid corresponded to the most hydrophobic sample (SI). On the other hand, protonation with sulfamic acid provided the most hydrophilic PANI salt particles (S4). The PANI base particles (S2) and the particles reprotonated with tartaric acid (S3) represent intermediate cases.

\subsection{Preparation of PANI particle suspensions}

PANI powders were ground in an agate mortar, sieved to obtain particle sizes smaller than $45 \mathrm{jam}$, and dried at $80{ }^{\circ} \mathrm{C}$ in a vacuum oven to constant weight. The suspensions (10 vol.\%) were prepared by mixing PANI powders with silicone oil (Lukosiol M200, Chemical Works Kolín, Czech Republic; viscosity $r j_{c}=200 \mathrm{mPa}$ s, density $p_{c}=$ $0.965 \mathrm{~g} \mathrm{crrT}^{3}$, conductivity $o_{\mathrm{c}}{ } 10 \sim^{\mathrm{n}} \mathrm{S} \mathrm{cm}^{-1}$ ). The suspensions were stirred at first mechanically, then in an ultrasonic bath for $30 \mathrm{~s}$ before ER measurement.

Table 1

The contact angles, 0 and densities, $p$ of PANI protonated in aqueous solutions of acids.

\begin{tabular}{|c|c|c|c|}
\hline PANהI sample & Aquegus acid & $\theta(0)$ & $\rho\left(\mathrm{gcm}^{-3}\right)$ \\
\hline$\$ 1$ & 5\% perfluorooctaresulfonic acid & 102 & 1.45 \\
\hline 52 & None & 88 & 1.33 \\
\hline 53 & 1 M tartaric acid & 51 & 1,35 \\
\hline 54 & 1 W sulfannic acid & 36 & 1.33 \\
\hline
\end{tabular}

\subsection{ER measurements of PANI particle suspensions}

Both the steady-flow measurements in the controlled shear rate mode and the dynamic oscillatory experiments in the linear viscoelasticity region were performed with a rotational rheometer Boh- lin Gemini (Malvern Instruments, UK), with parallel plates of $40 \mathrm{~mm}$ diameters and a gap of $0.5 \mathrm{~mm}$, modified for ER experiments. The plates were connected to a DC high-voltage source TREK 668B (TREK, USA) providing the electric field strength $E=0-500 \mathrm{Vmnrr}^{1}$. Before measurement at a new electric field strength, any structure produced by particles was destroyed by shearing the sample at the shear rate $20 \mathrm{~s}^{-1}$ for $80 \mathrm{~s}$. The temperature in all experiments was kept at $25{ }^{\circ} \mathrm{C}$.

\section{Results and discussion}

\subsection{Steady-state flow of PANI particle suspensions}

A considerable influence of hydrophobicity of particles on the flow behaviour of particle suspensions in the absence of electric field has been found (Fig. 1 ). Unlike the suspension of hydrophilic particles (S4) showing the pseudoplastic flow, the suspension of hydrophobic PANI (SI) was nearly Newtonian. Other PANI suspensions (S2, S3) showed slightly pseudoplastic behaviour. It appears that good compatibility of hydrophobic particles with the silicone oil provided a system of highly isolated particles with very weak interactions and high fluidity. In the contrast, the incompatibility of hydrophilic particles with the oil results in strong particle interactions which hinder their movement in the flow field. In this system also particle aggregation and formation of clusters may take place. As a result, the shear stress and viscosity of suspensions, especially at low shear rates, were high and the suspensions behaved as non-Newtonian materials. A similar behaviour was

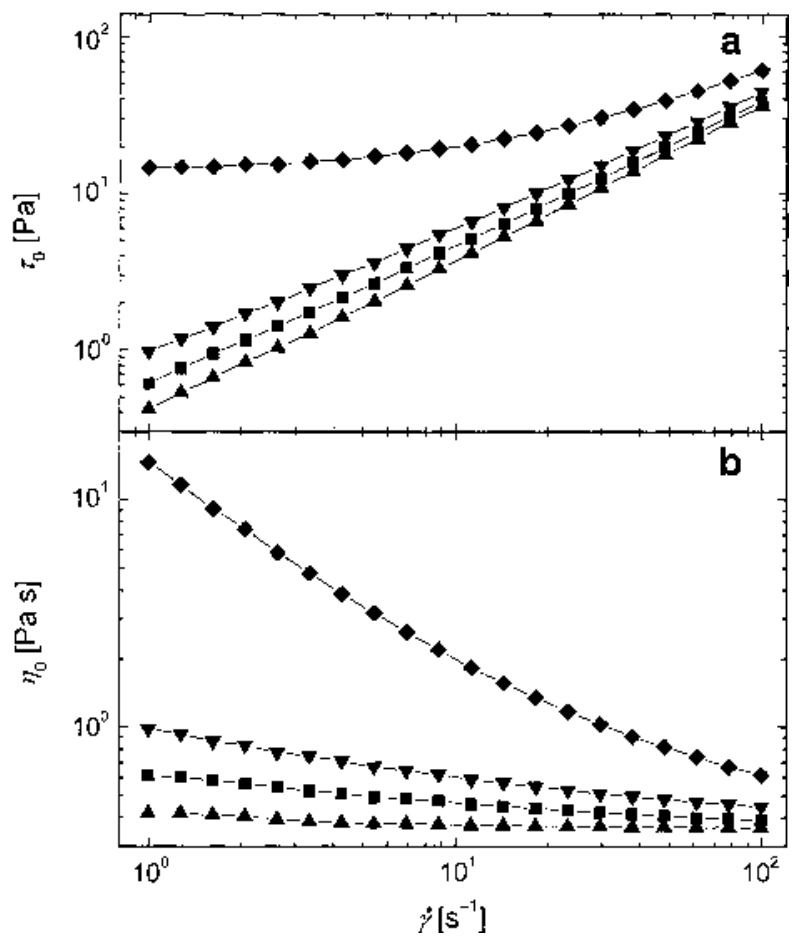

Fig. 1. The dependence of the shear stress, $\mathrm{t}_{\mathrm{l}}(\mathrm{a})$, and viscosity, $\left.y\right]_{q}(\mathrm{~b})$ of PANI suspensions on the shear rate, $y$ in the absence of electric field. PANI samples: $\triangle$ SI, - S2,T S3, + S4. 
observed for suspensions of silica particles in silicone oil and for particles treated with urea [10].

Under the influence of an electric field, the shear stress and the viscosity of suspensions of hydrophobic PANI (Fig. 2) increased. The slopes $1.9,1.4$ and 1.8 of the double logarithmic plot of the shear stress vs. electric field strength (Fig. 3) correspond to fully polarized non-interacting hydrophobic (SI), rather hydrophobic (S2) and rather hydrophilic (S3) particles, respectively. On the other hand, the slope 0.54 for the hydrophilic PANI salt (S4) suggests a low effect of polarization. Thus, the performance of these

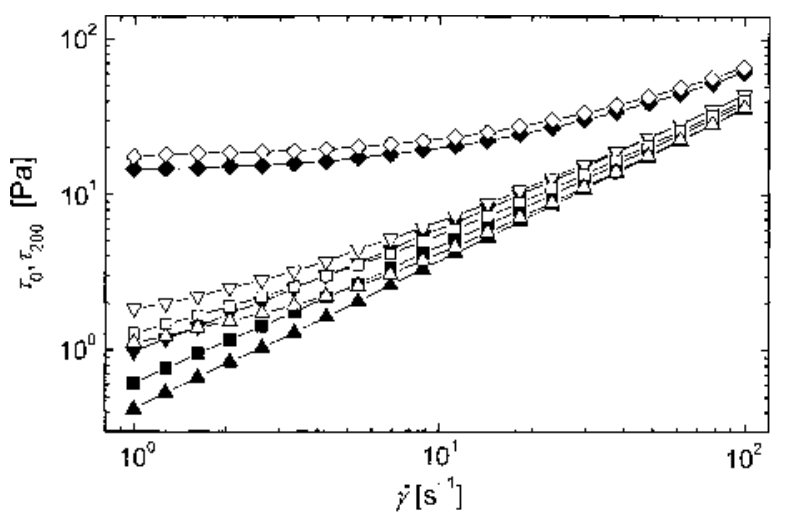

Fig. 2. The dependence of the shear stress, $x$ of PANI suspensions on the shear rate, $\}^{\prime}$. The electric field strength, $E\left(\mathrm{Vmm}^{-1}\right)$ : 0 (solid), 200 (open symbols). For the meaning of symbols, see Fig. 1.

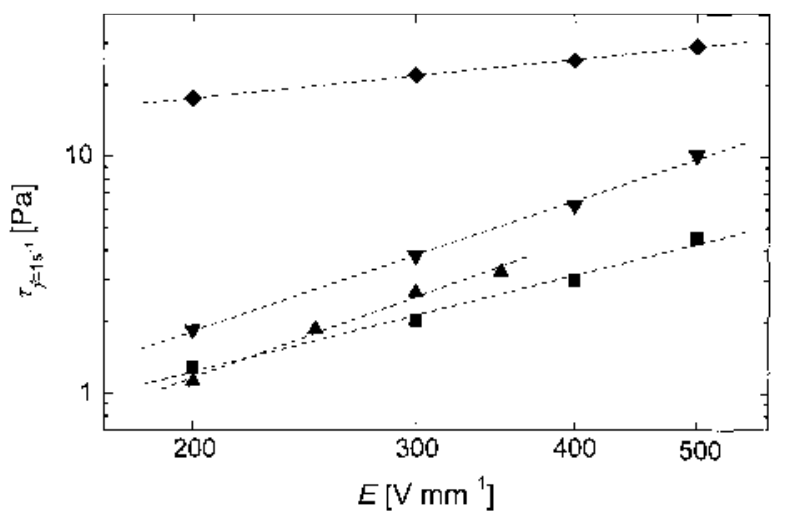

Fig. 3. The dependence of the shear stress, $x$ on the electric field strength, $E$ at the shear rate, $y=1 \mathrm{~s}^{-1}$. For the meaning of symbols, see Fig. 1.

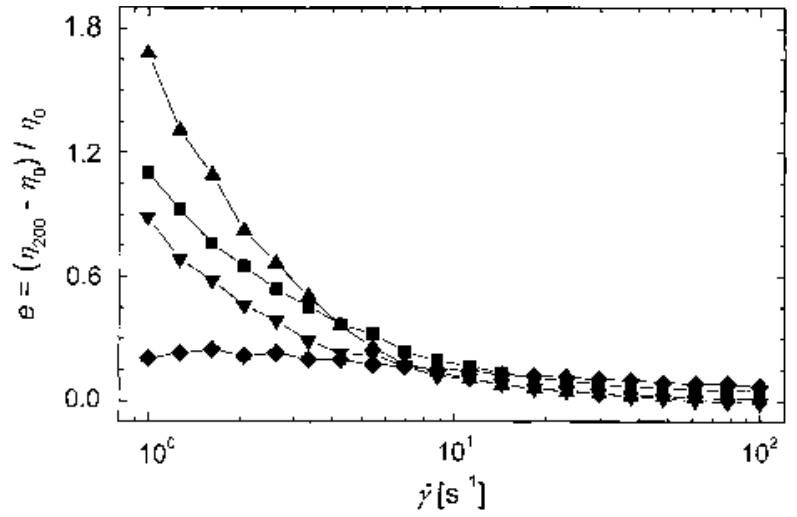

Fig. 4. The dependence of the relative increase in viscosity, $e=(/ / 200->70) />70$ on the shear rate, $y$. For the meaning of symbols, see Fig. 1. suspensions, $e=\left\{r j_{E^{-}} 77 \mathrm{o}\right) / * 7 \mathrm{o}$, is much higher than that of hydrophilic materials at low shear rates. However, when the hydrodynamic forces dominate over the electrostatic ones, at high shear rates, e inclines to the field-off state (Fig. 4).

3.2. The dynamic behaviour of PANI particle suspensions

A remarkable influence of hydrophobicity of particles on the dynamic behaviour of their suspensions characterizing the balance of

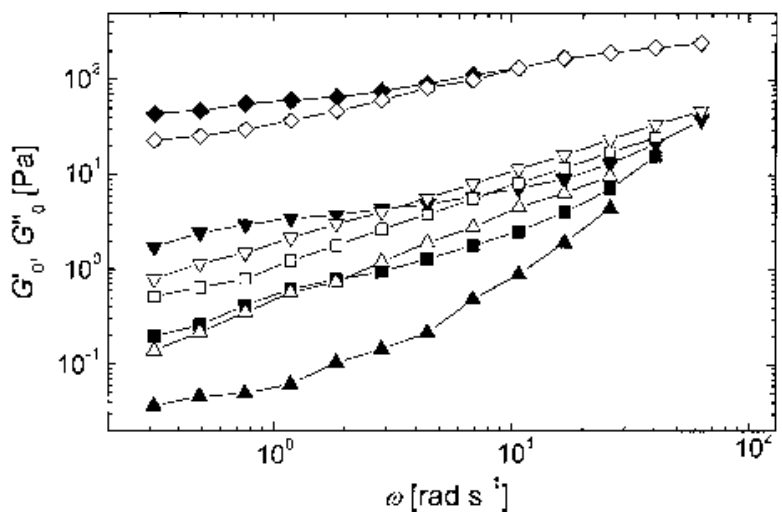

Fig. 5. The dependence of the field-off storage, $G_{0}^{\prime}($ solid), and loss, Gq moduli (open symbols) on the angular frequency, $\omega$. For the meaning of symbols, see Fig. 1.

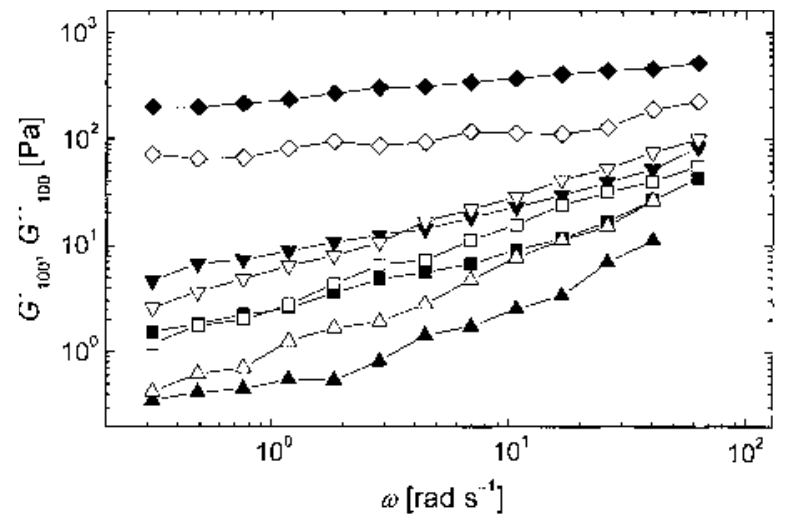

Fig. 6. The dependence of the storage, $G G_{w}^{\prime}$ (solid), and loss, $\mathrm{Gi}^{\prime}{ }^{\prime}$ moduli (open symbols) on the angular frequency, $\omega$ at the electric field strength, $E=100 \mathrm{~V} \mathrm{~mm}^{-1}$. For the meaning of symbols, see Fig. 1 .

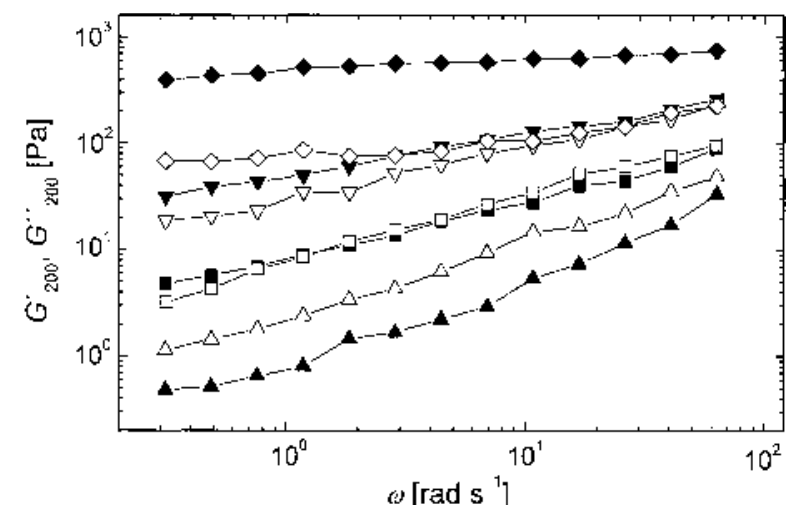

Fig. 7. The dependence of the storage, $G_{2 \infty}$ (solid), and loss, $\mathbf{G}_{\mathfrak{x}_{10}}$ moduli (open symbols) on the angular frequency, $c o$ at the electric field strength, $E=200 \mathrm{~V} \mathrm{~mm}^{-1}$. For the meaning of symbols, see Fig. 1. 
their fluidity and elasticity was observed. In the absence of electric field (Fig. 5), the loss modulus of the suspensions of hydrophobic (SI) and rather hydrophobic (S2) PANI particles was higher at low angular frequencies than the storage modulus $G^{\prime}$. This indicates a low elasticity of the system due to good compatibility of suspension particles with the oil. The elasticity of suspensions of rather hydrophilic particles (S3) was higher and, at low angular frequencies, the storage modulus dominated, while at higher angular frequencies it remained low. In the case of highly hydrophilic PANI salt (S4), the storage modulus of suspension prevailed, but at higher angular frequencies it coincided with the loss modulus.

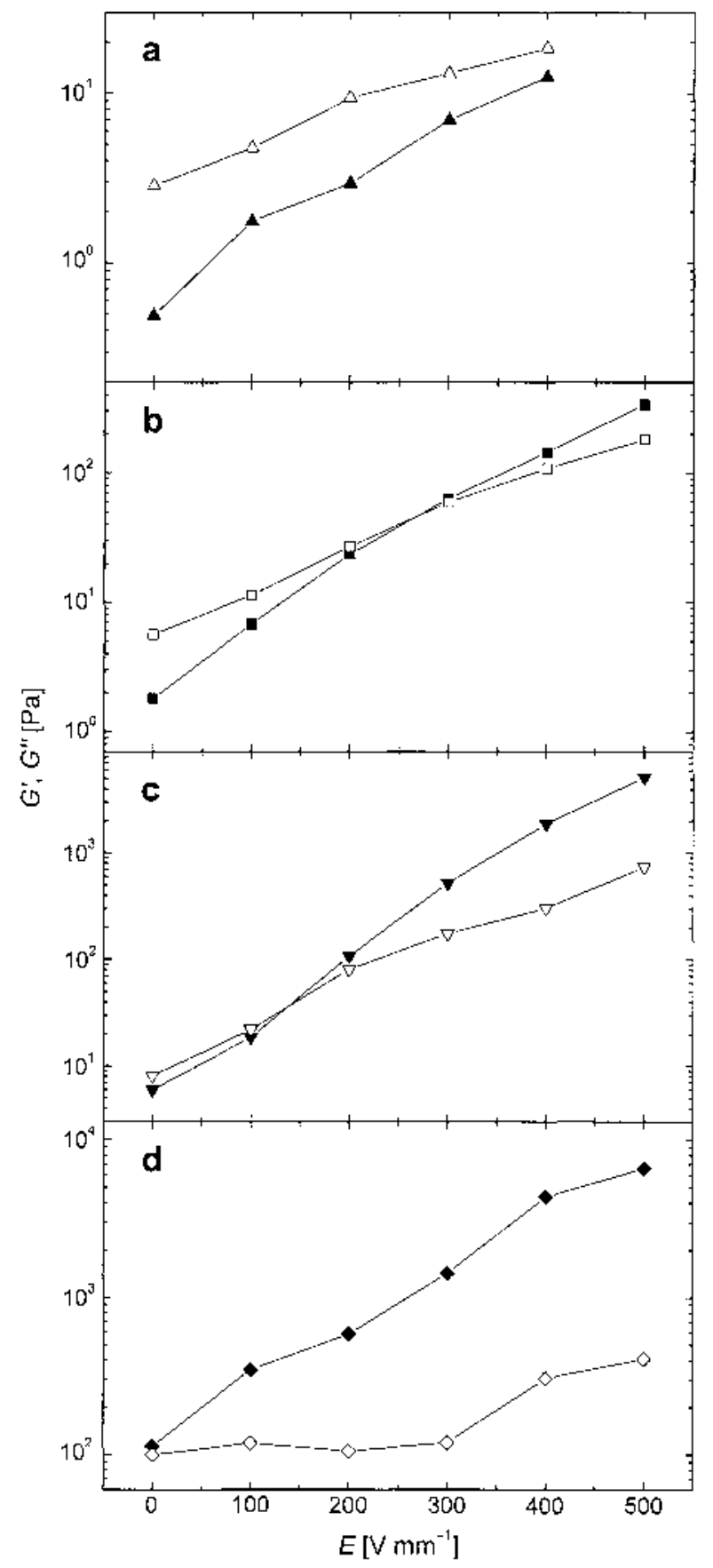

Fig. 8. The dependence of the storage, $G^{\prime}$ (solid), and loss, $G^{\prime \prime}$ moduli (open symbols) on the electric field strength, $E$ at the angular frequency, $c=7 \mathrm{rads}^{-1}$. For the meaning of symbols, see Fig. 1 .
When the electric field was applied, both moduli, in particular the storage component, increased due to formation of an organized structure of polarized particles with steeply increasing elasticity (Fig. 6). Thus, the storage modulus gradually dominated also in the suspensions of rather hydrophobic and hydrophilic particles (S2, S3), respectively. In the case of PANI salt (SI), the viscous character of suspensions prevails even in the presence of electric field due to weaker particle interactions (Fig. 7).

Fig. 8 illustrates a different dependence of viscoelastic moduli for particle suspensions of various hydrophobicity on the electric field strength at a single angular frequency $\left(c o=1 \mathrm{rad} \mathrm{s}^{-1}\right)$. For the most hydrophobic PANI (SI), the loss modulus prevailed up to the highest field strength used $\left(E=400 \mathrm{~V} \mathrm{~mm}^{-1}\right)$. The difference between the moduli diminished and, as observed on extrapolation to the highest field strengths, disappeared at $c a . E=500 \mathrm{~V} \mathrm{~mm}^{-1}$ (Fig. 8a). For rather hydrophobic (S2) and rather hydrophilic (S3) PANI samples, the dependences of the moduli cross at low field strengths as their hydrophilicity increases (Fig. 8b and c). In the case of hydrophilic PANI salt (S4), the predominance of the storage modulus in the whole range of the field strengths was observed as a consequence of strong particle interactions (Fig. 8d).

\section{Conclusions}

The results demonstrate that the compatibility of PANI suspension particles with the silicone oil depends on the hydrophilicity of the particles. The compatibility can significantly affect a relative increase in viscosity and elasticity of the material due to application of electric field. Thus, hydrophobic particles suspended in a hydrophobic silicone oil provide a homogeneous suspension with a low field-off viscosity. On application of electric field, a self-organized viscoelastic chain structure of independent polarized particles can arise and a fast increase in viscoelasticity sets in. On the other hand, due to interactions of hydrophilic particles, aggregates or clusters are formed and, consequently, the field-off suspension viscoelasticity is high. The effect of the electric field on a relative increase in this quantity is much lower than that in suspensions of hydrophobic particles. This means that the compatibility of suspension particles with the silicone oil is a decisive factor controlling the ER efficiency, and should be taken into account in the design of high-performance ER liquids.

\section{Acknowledgements}

The authors acknowledge the financial support of the Ministry of Education, Youth and Sports of the Czech Republic (MSM 7088352101), the Grant Agency of the Czech Republic (202/09/1626) and the Grant Agency of the Academy of Sciences of the Czech Republic (IAA 400500905).

\section{References}

[1] W.M. Winslow, US Patent 2417850 (1947).

[2] H. Block, J.P. Kelly, J. Phys. D Appl. Phys. 21 (1988) 1661.

[3] T.C. Jordan, M.T. Shaw, IEEE Trans. Electron. Insul. 24 (1998) 849.

[4] K.M. Blackwood, H. Block, Trends Polym. Sci. 14 (1993) 98.

[5] M. Parthasarathy, D.J. Klingenberg, Mater. Sci. Eng. R 17 (1996) 57.

[6] T. Hao, J. Colloid Interface Sci. 206 (1998) 240.

[7] T. Hao, A. Kawai, F. Ikazaki, J. Colloid Interface Sci. 239 (2001) 106.

[8] O. Quadrat, J. Stejskal, J. Ind. Eng. Chem. 12 (2006) 352.

[9] A. Lengálová, V. Pavlinek, P. Sáha, J. Stejskal, O. Quadrat, J. Colloid Interface Sci. 258 (2003) 174.

[10] T. Belza, V. Pavlinek, P. Sáha, O. Quadrat, Colloid Surf. A 316 (2007) 89.

[11] B.X. Wang, Y. Zhao, X.P. Zhao, Colloid Surf. A 295 (2007) 27.

[12] X. Huang, W. Wen, S. Yang, P. Sheng, Solid State Commun. 139 (2006) 581.

[13] Z. Zhang, Z. Wei, M. Wan, Macromolecules 35 (2002) 5937.

[14] I. Sapurina, J. Stejskal, Polym. Int. 57 (2008) 1295.

[15] J. Stejskal, J. Prokeš, M. Trchová, React. Funct. Polym. 68 (2008) 1355.

[16] H.J. Choi, T.W. Kim, M.S. Cho, S.G. Kim, M.S. Jhon, Eur. Polym. J. 33 (1997) 699.

[17] J.H. Lee, M.S. Cho, H.J. Choi, M.S. Jhon, Colloid Polym. Sci. 277 (1999) 73. 
[18] H.J. Choi, M.S. Cho, K. To, Physica A 254 (1998) 272

[19] H.J. Choi, L.H. Lee, M.S. Cho, M.S. Jhon, Polym. Eng. Sci. 39 (1999) 493

[20] M.S. Cho, Y.H. Cho, H.J. Choi, M.S. Jhon, Langmuir 19 (2003) 5875.
[21] M. Stenicka, V. Pavlinek, P. Saha, N.V. Blinova, J. Stejskal, 0. Quadrat, Colloid Polym. Sci. 287 (2009) 403.

[22] J. Stejskal, R.G. Gilbert, Pure Appl. Chem. 74 (2002) 857. 